\title{
Tumor PD-L1 expression, immune cell correlates and PD-1+ lymphocytes in sentinel lymph node melanoma metastases
}

Hojabr Kakavand ${ }^{1,2}$, Ricardo E Vilain ${ }^{1,3}$, James S Wilmott ${ }^{1,2}$, Hazel Burke ${ }^{1}$, Jennifer H Yearley ${ }^{4}$, John F Thompson ${ }^{1,2}$, Peter Hersey ${ }^{1,2,5}$, Georgina V Long ${ }^{1,2}$ and Richard A Scolyer ${ }^{1,2,3}$

${ }^{1}$ Melanoma Institute Australia, North Sydney, NSW, Australia; ${ }^{2}$ Sydney Medical School, The University of Sydney, Sydney, NSW, Australia; ${ }^{3}$ Tissue Pathology and Diagnostic Oncology, Royal Prince Alfred Hospital, Camperdown, NSW, Australia; ${ }^{4}$ Merck Research Laboratories, Palo Alto, CA, USA and ${ }^{5}$ Kolling Institute of Medical Research, Royal North Shore Hospital, St. Leonards, NSW, Australia

\begin{abstract}
Melanoma patients with sentinel lymph node metastases have variable 5-year survival rates (39-70\%). The prognostic significance of tumor-infiltrating lymphocytes in sentinel lymph node metastases from such patients is currently unknown. Anti-PD-1/PD-L1 inhibitors have significantly improved clinical outcome in unresectable AJCC stage IIIC/IV metastatic melanoma patients, and are being trialed in the adjuvant setting in advanced stage disease, however, their role in early stage (sentinel lymph node positive) metastatic disease remains unclear. The aims of this study were to characterize, in sentinel lymph nodes, the subpopulations of lymphocytes that interact with metastatic melanoma cells and analyze their associations with outcome, and to determine tumor PD-L1 expression as this may provide a rational scientific basis for the administration of adjuvant anti-PD-1/PD-L1 inhibitors in sentinel lymph node positive metastatic melanoma patients. Sentinel lymph nodes containing metastatic melanoma from 60 treatment-naive patients were analyzed for CD3, CD4, CD8, FOXP3, PD-1, and PD-L1 using immunohistochemistry on serial sections. The results were correlated with clinicopathologic features and outcome. Positive correlations between recurrence-free/overall survival with the number of CD3+ tumorinfiltrating lymphocytes (hazard ratio $=0.36(0.17-0.76), P=0.005$; hazard ratio $=0.29(0.14-0.61), P=0.0005$, respectively), the number of CD4+ tumor-infiltrating lymphocytes (hazard ratio $=0.34(0.15-0.77), P=0.007$; hazard ratio $=0.32(0.14-0.74), P=0.005$, respectively), and the number of CD8+ tumor-infiltrating lymphocytes (hazard ratio $=0.42(0.21-0.85), P=0.013$; hazard ratio $=0.32(0.19-0.78), P=0.006$, respectively) were observed. There was also a negative correlation with the number of peritumoral PD-1+ lymphocytes (hazard ratio = 2.67 (1.17-6.13), $P=0.016$; hazard ratio $=2.74(1.14-6.76), P=0.019$, respectively). Tumoral PD-L1 expression was present in 26 cases $(43 \%)$ but did not correlate with outcome. The findings suggest that T-cell subsets in sentinel lymph node metastases can predict melanoma patient outcome. In addition, the relatively high number of PD-L1 positive sentinel lymph node melanoma metastases provides a rationale for anti-PD-1 therapy trials in sentinel lymph node positive melanoma patients, particularly those with peritumoral PD-1+ lymphocytes.

Modern Pathology (2015) 28, 1535-1544; doi:10.1038/modpathol.2015.110; published online 25 September 2015
\end{abstract}

Sentinel lymph node biopsy has become the standard of care for accurately staging primary cutaneous melanomas $\geq 1 \mathrm{~mm}$ in Breslow thickness in most major melanoma treatment centers worldwide. ${ }^{1}$ Patients with a positive sentinel lymph node that

Correspondence: H Kakavand, BSc (Hons.), Royal Prince Alfred Hospital, R6.51, Level 6 Gloucester House, Camperdown, NSW 2050, Australia.

E-mail: hojabr.kakavand@melanoma.org.au

Received 24 May 2015; revised 11 August 2015; accepted 29

August 2015; published online 25 September 2015 undergo completion lymphadenectomy still have variable 5-year survival rates ranging from $39-70 \%$ depending on factors such as the number of tumorbearing nodes, sentinel lymph node tumor burden, presence or absence of primary tumor ulceration, and thickness of the primary melanoma. ${ }^{2}$ These patients may benefit from adjuvant therapies, which act to reduce the risk of relapse and dissemination to distant sites. Large randomized clinical trials have demonstrated a marginal benefit for adjuvant interferon, but associated significant toxicity has limited its role. ${ }^{3-6}$ 
Recent clinical trials have demonstrated that patients with unresectable AJCC stage IIIC \IV metastatic melanoma treated with drugs inhibiting CTLA-4 on T cells (ipilimumab), ${ }^{7}$ or the mitogenactivated protein kinase (MAPK) pathway in BRAF mutant melanoma (vemurafenib, dabrafenib, cobimetinib, and trametinib) have significantly improved progression-free survival and overall survival. ${ }^{8-12}$ Similarly, improvements in clinical outcomes have been achieved following treatment with monoclonal antibodies (MAb) that target the programmed cell death receptor 1 (PD-1) (also known as CD279) immune checkpoint pathway. ${ }^{13,14}$ Success in the metastatic setting with targeted therapies (BRAF and MEK inhibitors) and immunotherapies (CTLA-4, PD-1, and PD-L1 inhibitors) have prompted a number of ongoing phase III randomized clinical trials, as postoperative adjuvant therapies, assessing their efficacy in patients with high risk AJCC stage IIIB/C resectable metastatic melanoma (NCT01274338, NCT00636168, NCT01682083, NCT01667419, and NCT02362594). Whether they also have a role as adjuvant therapies in resected early stage (sentinel lymph node positive) metastatic melanoma is unknown.

PD-1 is expressed by a subset of activated T cells, as well as other immune cells ${ }^{15,16}$ and the binding of PD-1 on T cells with its ligands programmed cell death ligand 1 (PD-L1, CD274) 17,18 and programmed cell death ligand 2 (PD-L2, CD273) ${ }^{19,20}$ negatively regulates antigen receptor signaling and inhibits immune responses against PD-ligand expressing tumor cells and antigen-presenting cells. PD-L1 is expressed on subsets of $\mathrm{T}$ cells, B-cells, macrophages, and dendritic cells and may also be expressed by tumor cells thus facilitating a mechanism of escaping anti-tumor immunity. PD-L1 expression may be further upregulated by cytokines such as interferon gamma (IFN- $\gamma$ ) released by CD4+ helper $\mathrm{T}$ cells, ${ }^{21}$ which is believed to result in feedback suppression and evasion of immune responses against the tumor. ${ }^{22,23}$ We have previously demonstrated that there is an influx of CD4+ and CD8+ lymphocytes into melanomas of patients treated with BRAF inhibitor alone ${ }^{24}$ and more recently with the combination of BRAF and MEK inhibitors. ${ }^{25}$ It has also recently been shown that a higher density of peritumoral CD8+ lymphocytes at baseline is predictive of response in patients treated with PD-1 inhibitors. ${ }^{26}$

It is currently unknown whether PD-1/PD-L1 inhibitors will improve survival when administered in an adjuvant setting, and whether known predictors of response such as tumor PD-L1 expression ${ }^{13}$ and peritumoral CD8+ lymphocytes, ${ }^{26}$ which have been identified in advanced stage metastatic melanoma patients treated with PD-1 inhibitors, will also predict responses in the adjuvant setting. In this study, we sought to (1) characterize the extent and subpopulations (cytotoxic, helper, and regulatory) of tumor-infiltrating and peritumoral lymphocytes and determine their prognostic significance, and (2) decipher whether the PD-1/PD-L1 pathway contributes to inhibition of the immune system, in sentinel lymph node-positive melanoma patients. The study also sought to inform whether there is a scientific rationale for the use of anti-PD-1 inhibitors in patients with sentinel lymph node positive melanoma and to identify subgroups of patients who are most likely to receive benefit from such treatments.

\section{Materials and methods}

\section{Study Design and Patient Selection}

This study was undertaken at Melanoma Institute Australia and the Royal Prince Alfred Hospital, Sydney, Australia with Human Ethics Committee approval (X11-0289, HREC/11/RPAH/444). One hundred and ten patients who underwent a sentinel lymph node biopsy procedure at Melanoma Institute Australia and its' associated hospitals between 1993 and 2008 with pathologically identified metastatic melanoma in their sentinel lymph node were included in this study and, based on clinical follow up data, grouped into either 'developed recurrence' or 'no recurrence' groups matched for Breslow thickness, ulceration and maximum diameter of the metastasis in the sentinel lymph node. Of these, 60 sentinel lymph node biopsy samples were selected because they had tumor present in all the serial sections $(7 \times 4-\mu \mathrm{m}$ thick sections), as assessed by H\&E staining performed on the last slide. Patient demographics (age, gender), primary tumor characteristics (Breslow thickness, Clark level, ulceration, melanoma subtype), features of the sentinel lymph node biopsy (site of sentinel lymph node biopsy, number of positive sentinel lymph nodes, and maximum diameter of tumor), and results of the completion lymph node dissection were determined from review of clinical records in the Melanoma Institute Australia Melanoma Research Database. Recurrence-free survival and overall survival were calculated from date of the sentinel lymph node biopsy procedure to an event (either recurrence or death) or until last follow up (censored; Table 1).

\section{Immunohistochemistry}

All immunohistochemistry staining was performed on 4- $\mathrm{\mu m}$ thick sections using an Autostainer Plus (Dako-Agilent Technologies) with appropriate positive and negative controls. Sections were baked for $60 \mathrm{~min}$ at $60^{\circ} \mathrm{C}$ in a dehydration oven and heatinduced epitope retrieved in the PT link (DakoAgilent technologies) using EnVision FLEX target retrieval solution for $20 \mathrm{~min}$ at $97^{\circ} \mathrm{C}$ then cooled to room temperature in TBST Wash buffer for $5 \mathrm{~min}$. Slides were incubated with the following antibodies at the following dilutions: CD3 (Cell MarqueMRQ39) 1:500, CD4 (Cell Marque-SP35) 1:100, CD8 (Cell Marque-SP16) 1:200, PD-1 (Cell Marque 
Table 1 Clinical and pathological characteristics of the sentinel lymph node patients: $(n=60)$

\begin{tabular}{|c|c|}
\hline Parameter & Value (range) \\
\hline \multicolumn{2}{|l|}{ Sex } \\
\hline Male & 39 \\
\hline Female & 21 \\
\hline \multicolumn{2}{|l|}{ Age } \\
\hline Median (years) & $56(17-87)$ \\
\hline \multicolumn{2}{|l|}{ Primary melanoma features } \\
\hline Median Breslow thickness (mm) & $2.65(0.85-6.5)$ \\
\hline \multicolumn{2}{|l|}{ Subtype } \\
\hline Nodular & 34 \\
\hline Superficial spreading & 18 \\
\hline Acral & 4 \\
\hline Desmoplastic & 2 \\
\hline Lentigo maligna & 1 \\
\hline Nevoid & 1 \\
\hline \multicolumn{2}{|l|}{ Clark Level } \\
\hline III & 10 \\
\hline IV & 42 \\
\hline $\mathrm{V}$ & 8 \\
\hline \multicolumn{2}{|l|}{ Ulceration } \\
\hline Present & 31 \\
\hline Absent & 29 \\
\hline \multicolumn{2}{|l|}{ Sentinel lymph node biopsy features } \\
\hline Median no. of positive SLNs & $1(1-6)$ \\
\hline Median max diameter of metastasis (mm) & $2.76(0.95-18)$ \\
\hline \multicolumn{2}{|l|}{ Site } \\
\hline Neck & 8 \\
\hline Axillae & 27 \\
\hline Groin & 25 \\
\hline \multicolumn{2}{|l|}{ Complete lymph node dissection features $(n=47)$} \\
\hline Median no. of nodes removed & $21(4-73)$ \\
\hline \multicolumn{2}{|l|}{ Complete lymph node dissection status: } \\
\hline Positive & 10 \\
\hline Negative & 37 \\
\hline Median no. of positive nodes & $1(1-12)$ \\
\hline \multicolumn{2}{|l|}{ Follow up } \\
\hline Median recurrence-free survival (months) & $24(1-161)$ \\
\hline Median overall survival (months) & $38(2-186)$ \\
\hline \multicolumn{2}{|l|}{ Site of recurrence $(\mathrm{n}=36)$ : } \\
\hline Multiple & 24 \\
\hline Lymph node only & 0 \\
\hline In transit only & 2 \\
\hline Visceral only & 10 \\
\hline
\end{tabular}

-MRQ-22/NAT105) 1:100, FOXP3 (AbcamAB22510) 1:200 and PD-L1 (Merck-22C3) 1:1000. The Envision flex Mouse linker (K8022) was used to amplify the signal for PD-1 and PD-L1. Antibody detection utilized DAB chromagen for visualization according to the manufacturer's instructions (Dako). Slides were then counterstained with hematoxylin.

\section{Assessment of Tumor PD-L1 and Lymphocyte Subsets}

Digital copies of all immunohistochemistry slides were made using the Nanozoomer 2.0 HT (Hammamatsu) whole slide scanner at $\times 20$ magnification. For assessment of PD-L1 expression, the percentage of tumor cells demonstrating positive

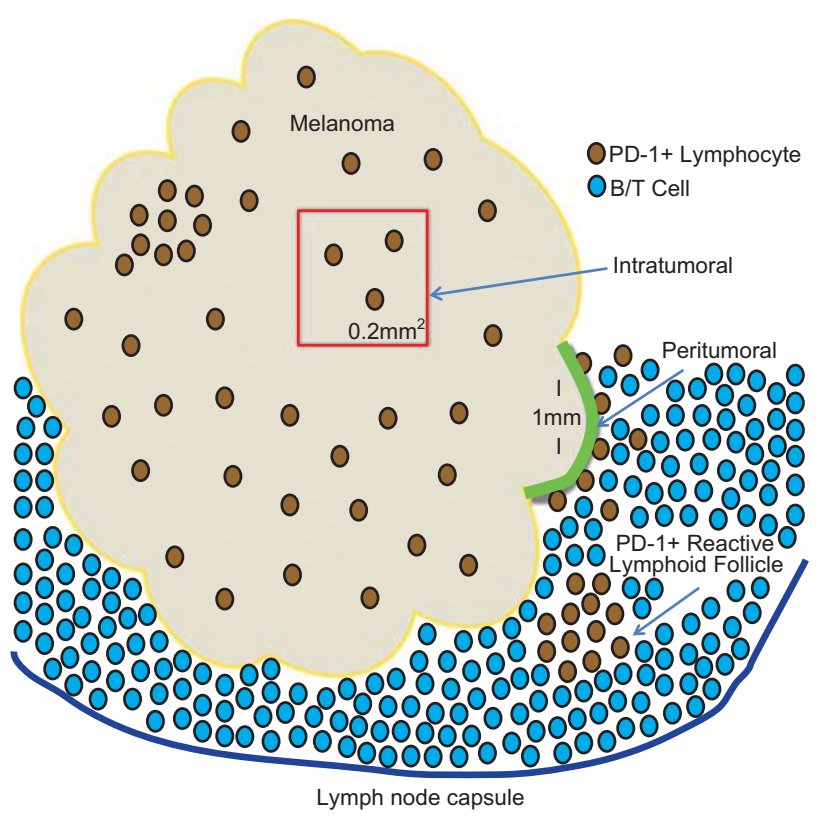

Figure 1 A schematic figure demonstrating a sentinel lymph node with metastatic melanoma. The number of immunoreactive lymphocytes for each of the immune markers was counted in the $1 \times 0.02 \mathrm{~mm}$ peritumoral area (green line) at the interface between the tumor and the lymph node parenchyma. The number of immunoreactive lymphocytes for each of the immune markers was counted in the $0.2 \mathrm{~mm}^{2}$ intratumoral area (red box) within the tumor deposit. The example demonstrated is for PD-1+ lymphocytes.

cytoplasmic membrane staining was determined and the intensity of staining was scored utilizing a semiquantitative scale ( $0-3+)$ : no staining (0), weakly positive staining $(1+)$, moderately positive staining $(2+)$, and strongly positive staining $(3+)$. Positive PD-L1 tumor staining was defined as $\geq 1 \%$ tumor cell expression. Digitally scanned CD3-stained tissue sections were used to generate semiquantitative cell counts within two distinct sentinel lymph node compartments; (1) The peritumoral region, defined as the junction between tumor and immune stroma along a $1.0 \times 0.02 \mathrm{~mm}$ delimited zone, which whenever possible was selected to be located adjacent to the tumor periphery exhibiting the strongest level of PD-L1 expression; and (2) The intratumoral region, defined by a $0.2 \mathrm{~mm}^{2}$ bounded area containing an immune infiltrate considered to be most representative of the overall intratumoral lymphocytic density (Figure 1). The CD3-stained sections defined the intratumoral and peritumoral regions analyzed in serially cut sections stained for other T-cell subsets (CD4, CD8, PD-1, and FOXP3). The slides were reviewed and assessed by two observers (RV and HK).

\section{Statistical Analysis}

Statistical analyses were conducted with 'PASW Statistics 21' SPSS, IBM. Bivariate correlations 

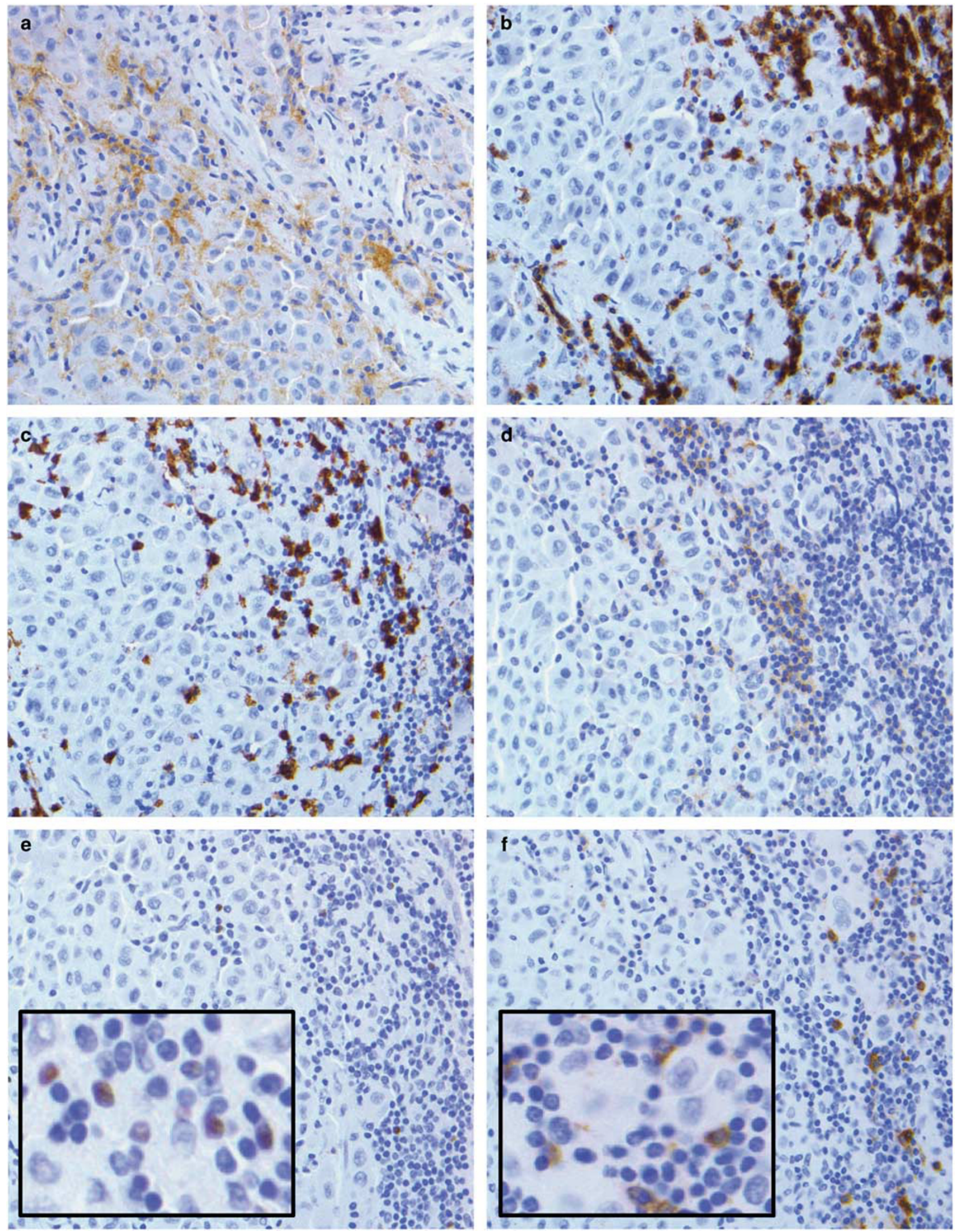

Figure 2 Immunohistochemistry undertaken on serial sections from a sentinel lymph node biopsy containing metastatic melanoma. The expression of membranous tumor PD-L1 (a), CD3+ (b), CD4+ helper (c), CD8+ cytotoxic (d), FOXP3+ regulatory (e), and PD-1+ differentiated (f) lymphocytes at $\times 200$ magnification is demonstrated. High-power inserts demonstrate intratumoral FOXP3+ and PD-1+ lymphocyte staining. 
between the different immune markers and tumor PD-L1 expression were conducted using the Spearman's rho test. Univariate survival analysis was carried out using the Kaplan-Meier method together with the log-rank (Mantel-Cox) test to calculate statistical significance. Cox regression analysis was used to generate univariate hazard ratios, 95\% confidence intervals, and corresponding $P$ values. Statistical significance was defined as a probability level $<0.05$.

\section{Results}

\section{Patients and Biopsies}

Sixty sentinel lymph node biopsy specimens were available for analysis. There were from 39 male and 21 female patients with a median age of 56 years (range $=17-87$ ). In the 60 patients, the primary melanoma was ulcerated in $52 \%(n=31)$ of cases, and the median Breslow thickness was $2.65 \mathrm{~mm}$ (range $=0.85-6.5 \mathrm{~mm}$ ). The breakdown of the primary melanoma subtypes were 34 nodular, 18 superficial spreading, 4 acral, 2 desmoplastic, 1 lentigo maligna, and 1 nevoid melanoma. The median number of positive sentinel lymph nodes was 1 (range $=1-6$ ) and the median maximum diameter of metastatic melanoma present in the sentinel lymph nodes was $2.76 \mathrm{~mm}$ (range $=$ 0.95-18 mm). There were 47 sentinel lymph node positive patients who subsequently elected to have a completion lymph node dissection, of whom 10 had metastatic melanoma present in one or more non-sentinel lymph nodes, with a median number of 1 positive non-sentinel node (range $=1-12$; Table 1).

\section{Tumor PD-L1 and Immune Correlates}

The membranous expression of tumor PD-L1 was highly heterogeneous with 26 (43\%) samples showing expression in $\geq 1 \%$ tumor cell with a median percentage of tumor cells positive in positive cases of $4 \%$ (range $=0-70 \%$ in all samples; Figure 2a, Table 2). The staining intensity of PD-L1 expression varied from negative (0) to strongly positive (3+). The immune markers CD3, CD4, CD8 and PD-1 showed membranous staining in lymphocytes, whereas FOXP3 was expressed within the nucleus of lymphocytes (Figures 2b-f, Table 2). All immune markers showed positive immune-reactivity, both at the interface between the tumor and the normal lymph node (peritumoral), and within the tumor deposit (intratumoral). Tumor PD-L1 expression significantly correlated with the presence of intratumoral CD3+ lymphocytes $(R=0.329, \quad P=0.011)$, peritumoral and intratumoral CD8+ lymphocytes $(R=0.256, P=0.048 ; R=0.448, P=0.0003$, respectively), peritumoral and intratumoral PD-1+ lymphocytes $(R=0.311, \quad P=0.016 ; \quad R=0.425, \quad P=0.001$,
Table 2 Median \% tumor PD-L1 expression and intratumoral and peritumoral lymphocyte counts

\begin{tabular}{lcc}
\hline Variable & Median (n=60) & Range \\
\hline \% Tumor PD-L1 & 0 & $0-70$ \\
Peritumoral PD-1 lymphocytes & 4 & $0-56$ \\
Intratumoral PD-1 lymphocytes & 3 & $0-138$ \\
Peritumoral CD3 lymphocytes & 66 & $23-193$ \\
Intratumoral CD3 lymphocytes & 106 & $0-776$ \\
Peritumoral CD4 lymphocytes & 10 & $0-55$ \\
Intratumoral CD4 lymphocytes & 14 & $0-77$ \\
Peritumoral CD8 lymphocytes & 34 & $9-119$ \\
Intratumoral CD8 lymphocytes & 59 & $0-572$ \\
Peritumoral FOXP3 lymphocytes & 14 & $0-49$ \\
Intratumoral FOXP3 lymphocytes & 21 & $0-146$ \\
\hline
\end{tabular}

respectively), and peritumoral and intratumoral FOXP3+ lymphocytes $(R=0.351, P=0.006 ; R=0.395$, $P=0.002$, respectively; Table 3 ). Using nonparametric analysis, patients with positive tumor PD-L1 expression had a larger tumor diameter in their sentinel lymph node biopsy (Mann-Whitney $\mathrm{U}-P=0.005$ ). There were no significant associations between percentage and intensity of tumor PD-L1 or the immune cell markers with age, sex, number of positive nodes, Breslow thickness, or ulceration of the primary melanoma.

\section{Recurrence-Free and Overall Survival}

At the time of last follow up (October 2014) the median recurrence-free survival was 24 months (range $=1-161$ months) and $36(60 \%)$ patients had experienced disease recurrence. The median overall survival at last follow up was 38 months (range $=$ 2-186 months). Twenty-four (40\%) patients were still alive, of whom 23 were alive with no sign of recurrence and one was alive with disease status unknown. Cox proportional hazard ratio modeling was used to determine optimal cutoff for expression of the various markers for prediction of recurrencefree survival and overall survival. There was no correlation observed between PD-L1 expression and either overall survival or recurrence-free survival in this cohort of patients (Table 4). A positive correlation was observed between higher $(>39.5)$ intratumoral CD3+ lymphocyte count and longer recurrence-free survival and overall survival (hazard ratio $=0.36(0.17-0.76), P=0.005$; hazard ratio $=0.29$ (0.14-0.61), $P=0.0005$, respectively; Figures $3 \mathrm{a}$ and b, Table 4). There was also a positive correlation observed between higher (>24) intratumoral CD4+ lymphocyte count and longer recurrence-free survival and overall survival (hazard ratio $=0.34(0.15-$ 0.77), $P=0.007$; hazard ratio $=0.32(0.14-0.74)$, $P=0.005$, respectively; Figures $3 \mathrm{c}$ and d,Table 4) and a positive correlation between the presence of higher (>29) intratumoral CD8+ lymphocyte count and longer recurrence-free survival and overall 
Table 3 Correlations between tumor PD-L1 and lymphocyte markers

\begin{tabular}{|c|c|c|c|c|c|c|c|c|c|c|c|}
\hline Spearman's Rho & $\% P D-L 1$ & $\begin{array}{c}\text { PD1 } \\
\text { (Peritumoral) }\end{array}$ & $\begin{array}{c}P D 1 \\
\text { (Intratumoral) }\end{array}$ & $\begin{array}{c}\text { CD3 } \\
\text { (Peritumoral) }\end{array}$ & $\begin{array}{c}C D 3 \\
\text { (Intratumoral) }\end{array}$ & $\begin{array}{c}\text { CD4 } \\
\text { (Peritumoral) }\end{array}$ & $\begin{array}{c}\text { CD4 } \\
\text { (Intratumoral) }\end{array}$ & $\begin{array}{c}\text { CD8 } \\
\text { (Peritumoral) }\end{array}$ & $\begin{array}{c}C D 8 \\
\text { (Intratumoral) }\end{array}$ & $\begin{array}{c}\text { FOXP3 } \\
\text { (Peritumoral) }\end{array}$ & $\begin{array}{c}\text { FOXP3 } \\
\text { (Intratumoral) }\end{array}$ \\
\hline \multicolumn{12}{|l|}{$\% P D-L 1$} \\
\hline Coefficient $(x)$ & & $0.311^{\mathrm{a}}$ & $0.425^{\mathrm{b}}$ & 0.164 & $0.329^{\mathrm{a}}$ & 0.209 & 0.155 & $0.256^{\mathrm{a}}$ & $0.448^{\mathrm{b}}$ & $0.351^{\mathrm{b}}$ & $0.395^{\mathrm{b}}$ \\
\hline Sig. (2-tailed) & & 0.016 & 0.001 & 0.214 & 0.011 & 0.112 & 0.241 & 0.048 & 0.000 & 0.006 & 0.002 \\
\hline \multicolumn{12}{|l|}{ PD1 (Peritumoral) } \\
\hline Correlation $(x)$ & $0.311^{\mathrm{a}}$ & & $0.584^{b}$ & 0.163 & 0.141 & $0.384^{\mathrm{b}}$ & 0.154 & $0.343^{\mathrm{b}}$ & 0.220 & $0.459^{\mathrm{b}}$ & 0.224 \\
\hline Sig. (2-tailed) & 0.016 & & 0.000 & 0.217 & 0.287 & 0.003 & 0.243 & 0.007 & 0.092 & 0.000 & 0.088 \\
\hline \multicolumn{12}{|l|}{ PD1 (Intratumoral) } \\
\hline Coefficient (x) & $0.425^{\mathrm{b}}$ & $0.584^{\mathrm{b}}$ & & $0.288^{\mathrm{a}}$ & $0.632^{\mathrm{b}}$ & 0.226 & $0.315^{\mathrm{a}}$ & $0.387^{\mathrm{b}}$ & $\mathbf{0 . 6 9 6}^{\mathrm{b}}$ & $0.431^{\mathrm{b}}$ & $0.658^{b}$ \\
\hline Sig. (2-tailed) & 0.001 & 0.000 & & 0.029 & 0.000 & 0.088 & 0.016 & 0.002 & 0.000 & 0.001 & 0.000 \\
\hline \multicolumn{12}{|l|}{ CD3 (Peritumoral) } \\
\hline Coefficient $(\mathrm{x})$ & 0.164 & 0.163 & $0.288^{\mathrm{a}}$ & & $0.441^{\mathrm{b}}$ & $0.361^{\mathrm{b}}$ & 0.247 & $\mathbf{0 . 6 9 8}^{\mathrm{b}}$ & $0.364^{\mathrm{b}}$ & $0.438^{\mathrm{b}}$ & $0.326^{\mathrm{a}}$ \\
\hline Sig. (2-tailed) & 0.214 & 0.217 & 0.029 & & 0.000 & 0.005 & 0.062 & 0.000 & 0.005 & 0.001 & 0.013 \\
\hline \multicolumn{12}{|l|}{ CD3 (Intratumoral) } \\
\hline Coefficient (x) & $0.329^{\mathrm{a}}$ & 0.141 & $0.632^{\mathrm{b}}$ & $0.441^{\mathrm{b}}$ & & 0.162 & $0.436^{\mathrm{b}}$ & $0.265^{\mathrm{a}}$ & $0.928^{\mathrm{b}}$ & 0.221 & $0.721^{\mathrm{b}}$ \\
\hline Sig. (2-tailed) & 0.011 & 0.287 & 0.000 & 0.000 & & 0.224 & 0.001 & 0.043 & 0.000 & 0.096 & 0.000 \\
\hline \multicolumn{12}{|l|}{ CD4 (Peritumoral) } \\
\hline Coefficient $(\mathrm{x})$ & 0.209 & $0.384^{\mathrm{b}}$ & 0.226 & $0.361^{\mathrm{b}}$ & 0.162 & & $\mathbf{0 . 5 9 6}^{\mathrm{b}}$ & 0.154 & 0.120 & 0.181 & 0.183 \\
\hline Sig. (2-tailed) & 0.112 & 0.003 & 0.088 & 0.005 & 0.224 & & 0.000 & 0.245 & 0.366 & 0.174 & 0.170 \\
\hline \multicolumn{12}{|c|}{ CD4 (Intratumoral) } \\
\hline Coefficient (x) & 0.155 & 0.154 & $0.315^{\mathrm{a}}$ & 0.247 & $0.436^{\mathrm{b}}$ & $0.596^{\mathrm{b}}$ & & 0.032 & $0.391^{\mathrm{b}}$ & 0.173 & $0.437^{\mathrm{b}}$ \\
\hline Sig. (2-tailed) & 0.241 & 0.243 & 0.016 & 0.062 & 0.001 & 0.000 & & 0.812 & 0.002 & 0.194 & 0.001 \\
\hline \multicolumn{12}{|l|}{ CD8 (Peritumoral) } \\
\hline Coefficient $(\mathrm{x})$ & $0.256^{\mathrm{a}}$ & $0.343^{\mathrm{b}}$ & $0.387^{\mathrm{b}}$ & $0.698^{\mathrm{b}}$ & $0.265^{\mathrm{a}}$ & 0.154 & 0.032 & & $0.338^{\mathrm{b}}$ & $0.493^{\mathrm{b}}$ & 0.219 \\
\hline Sig. (2-tailed) & 0.048 & 0.007 & 0.002 & 0.000 & 0.043 & 0.245 & 0.812 & & 0.008 & 0.000 & 0.096 \\
\hline \multicolumn{12}{|l|}{ CD8 (Intratumoral) } \\
\hline Coefficient (x) & $0.448^{\mathrm{b}}$ & 0.220 & $0.696^{\mathrm{b}}$ & $0.364^{\mathrm{b}}$ & $0.928^{\mathrm{b}}$ & 0.120 & $0.391^{\mathrm{b}}$ & $0.338^{\mathrm{b}}$ & & $0.302^{\mathrm{a}}$ & $0.771^{b}$ \\
\hline Sig. (2-tailed) & 0.000 & 0.092 & 0.000 & 0.005 & 0.000 & 0.366 & 0.002 & 0.008 & & 0.020 & 0.000 \\
\hline \multicolumn{12}{|c|}{ FOXP3 (Peritumoral) } \\
\hline Coefficient $(\mathrm{x})$ & $0.351^{\mathrm{b}}$ & $0.459^{\mathrm{b}}$ & $0.431^{\mathrm{b}}$ & $0.438^{\mathrm{b}}$ & 0.221 & 0.181 & 0.173 & $0.493^{\mathrm{b}}$ & $0.302^{\mathrm{a}}$ & & $0.520^{b}$ \\
\hline Sig. (2-tailed) & 0.006 & 0.000 & 0.001 & 0.001 & 0.096 & 0.174 & 0.194 & 0.000 & 0.020 & & 0.000 \\
\hline \multicolumn{12}{|c|}{ FOXP3 (Intratumoral) } \\
\hline Coefficient $(\mathrm{x})$ & $0.395^{\mathrm{b}}$ & 0.224 & $0.658^{\mathrm{b}}$ & $0.326^{\mathrm{a}}$ & $0.721^{\mathrm{b}}$ & 0.183 & $0.437^{\mathrm{b}}$ & 0.219 & $0.771^{\mathrm{b}}$ & $0.520^{\mathrm{b}}$ & \\
\hline Sig. (2-tailed) & 0.002 & 0.088 & 0.000 & 0.013 & 0.000 & 0.170 & 0.001 & 0.096 & 0.000 & 0.000 & \\
\hline
\end{tabular}

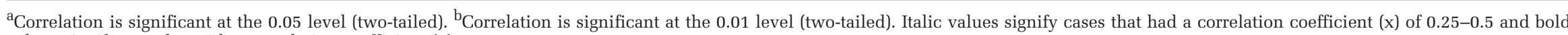
values signify samples with a correlation coefficient $(\mathrm{x})>0.5$. 
Table 4 Correlation between immunohistochemistry and clinical outcome measures

\begin{tabular}{|c|c|c|c|c|c|c|c|}
\hline Recurrence-free survival & Cut-off & Hazard ratio & Significance & Overall Survival & Cut-off & Hazard ratio & Significance \\
\hline \% PD-L1 & 0.5 & $1.42(0.73-2.76)$ & $P=0.3$ & PD-L1 & 0.5 & $1.36(0.7-2.64)$ & $P=0.37$ \\
\hline PD-1 Peritumoral & 1.5 & $2.67(1.17-6.13)$ & $P=0.016$ & PD-1 Peritumoral & 1.5 & $2.76(1.14-6.68)$ & $P=0.019$ \\
\hline PD-1 Intratumoral & 23.5 & $1.56(0.7-3.47)$ & $P=0.27$ & PD-1 Intratumoral & 0.5 & $0.74(0.36-1.5)$ & $P=0.4$ \\
\hline CD3 Peritumoral & 80 & $1.76(0.81-3.8)$ & $P=0.15$ & CD3 Peritumoral & 80 & $1.73(0.8-3.77)$ & $P=0.16$ \\
\hline CD3 Intratumoral & 39.5 & $0.36(0.17-0.76)$ & $P=0.005$ & CD3 Intratumoral & 39.5 & $0.29(0.14-0.61)$ & $P=0.00049$ \\
\hline CD4 Peritumoral & 25.5 & $0.53(0.2-1.36)$ & $P=0.18$ & CD4 Peritumoral & 21.5 & $0.65(0.3-1.4)$ & $P=0.27$ \\
\hline CD4 Intratumoral & 24 & $0.34(0.15-0.77)$ & $P=0.007$ & CD4 Intratumoral & 24 & $0.32(0.14-0.74)$ & $P=0.0053$ \\
\hline CD8 Peritumoral & 45.5 & $1.87(0.87-4.04)$ & $P=0.1$ & CD8 Peritumoral & 32.5 & $1.69(0.85-3.34)$ & $P=0.13$ \\
\hline CD8 Intratumoral & 29 & $0.42(0.21-0.85)$ & $P=0.013$ & CD8 Intratumoral & 29 & $0.39(0.19-0.78)$ & $P=0.0057$ \\
\hline FOXP3 Peritumoral & 7.5 & $2.31(0.87-6.15)$ & $P=0.085$ & FOXP3 Peritumoral & 7.5 & $2.25(0.85-5.97)$ & $P=0.096$ \\
\hline FOXP3 Intratumoral & 15.5 & $0.6(0.31-1.17)$ & $P=0.13$ & FOXP3 Intratumoral & 15.5 & $0.58(0.3-1.14)$ & $P=0.11$ \\
\hline
\end{tabular}

The bold entries signify the markers for which there was a significant association $(P<0.05)$ with progression free and overall survival.

survival (hazard ratio $=0.42(0.21-0.85), P=0.013$; hazard ratio $=0.32(0.19-0.78), P=0.006$, respectively) (Figures $3 \mathrm{e}$ and f, Table 4). Interestingly, there was a negative correlation between the higher (>1.5) peritumoral PD-1+ lymphocyte count and longer recurrence-free survival and overall survival (hazard ratio $=2.67(1.17-6.13), P=0.016$; hazard ratio $=2.74 \quad(1.14-6.76), \quad P=0.019, \quad$ respectively; Figures $3 \mathrm{~g}$ and $\mathrm{h}$, Table 4). There was no significant association between tumoral PD-L1 expression and recurrence-free survival or overall survival. A trend towards a negative correlation between higher (>7.5) peritumoral FOXP3+ lymphocyte count and longer recurrence-free survival and overall survival was observed, but this did not reach significance (hazard ratio $=2.31(0.87-6.15), P=0.085$; hazard ratio $=2.25 \quad(0.85-5.97), \quad P=0.096, \quad$ respectively; Table 4).

\section{Discussion}

Sentinel lymph node biopsy has become the standard of care for accurately staging melanoma patients with primary tumors $>1 \mathrm{~mm}$ Breslow thickness and it provides important prognostic information. ${ }^{1,27}$ Approximately $40 \%$ of patients with a positive sentinel lymph node will develop disease recurrence within 5 years and most of them will eventually die of melanoma. ${ }^{2}$ Adjuvant therapies have the potential to improve the outcome for these patients. Phase III clinical trial of adjuvant antiCTLA-4 inhibitor at higher doses than administered in the metastatic setting has also proven to be effective in improving recurrence-free survival in patients at high risk of recurrence compared with placebo. ${ }^{28}$ Given the efficacy of anti-PD-1/PD-L1 antibodies in patients with advanced stage melanoma, these therapies may also provide benefit in sentinel lymph node positive patients. To the best of our knowledge this is the first study that has evaluated tumor PD-L1 expression, a known biomarker of response to anti-PD-1 inhibitors in advanced stage melanoma, and other immune markers in positive sentinel lymph nodes of melanoma patients. We sought to identify the phenotype of the tumor and immune cells at the tumor-immune stroma interface and within the tumor deposit in an attempt to provide a rationale for the use of anti-PD-1/PD-L1 checkpoint inhibitor therapy in the adjuvant setting for sentinel lymph node positive melanoma patients.

The challenge that faces clinicians who now have an assortment of treatments available for patients with metastatic melanoma is the identification of predictive biomarkers that will help to determine the greatest likelihood of responses to the already considerable array of available targeted and immune therapies. The membranous expression of tumor PD-L1 is heterogeneous both within any given melanoma and between various samples from the same patient. ${ }^{29-31}$ Despite this evidence, tumoral PD-L1 expression had been demonstrated to be predictive of response to anti-PD-1 inhibitors. ${ }^{32,33}$ Nevertheless, recent studies have shown that some patients with 'negative' PD-L1 expression still have objective responses to anti-PD-1 inhibitors $^{32}$ and anti-PD-L1 inhibitors; ${ }^{34}$ therefore, reportedly absent PD-L1 expression cannot be used to exclude patients from this form of therapy. In addition, patients with PD-L1 negative tumors treated with the PD-1 antibody nivolumab had an improved overall survival compared with both patients, classified as PD-L1 positive and negative, treated with chemotherapy. ${ }^{32}$ A recent study reported that peritumoral CD8+ lymphocytes were the most predictive of response to anti-PD-1 inhibitors. ${ }^{26}$ In our cohort of patients, the expression of tumor PD-L1 correlated highly with the presence of intratumoral lymphocyte subsets, including those that were PD-1 positive. These results support previously reported findings by our group and others, ${ }^{25,26,29}$ which suggest the presence of lymphocytes that secrete IFN- $\gamma$, as well as other co-contributing factors, drives the expression of tumor PD-L1 in an acquired adaptive immune evasion phenomenon. This model is also supported by our finding that patients with positive tumor PD-L1 have a higher median diameter of tumor in their sentinel lymph node compared to patients with tumors that were negative for PD-L1. This 

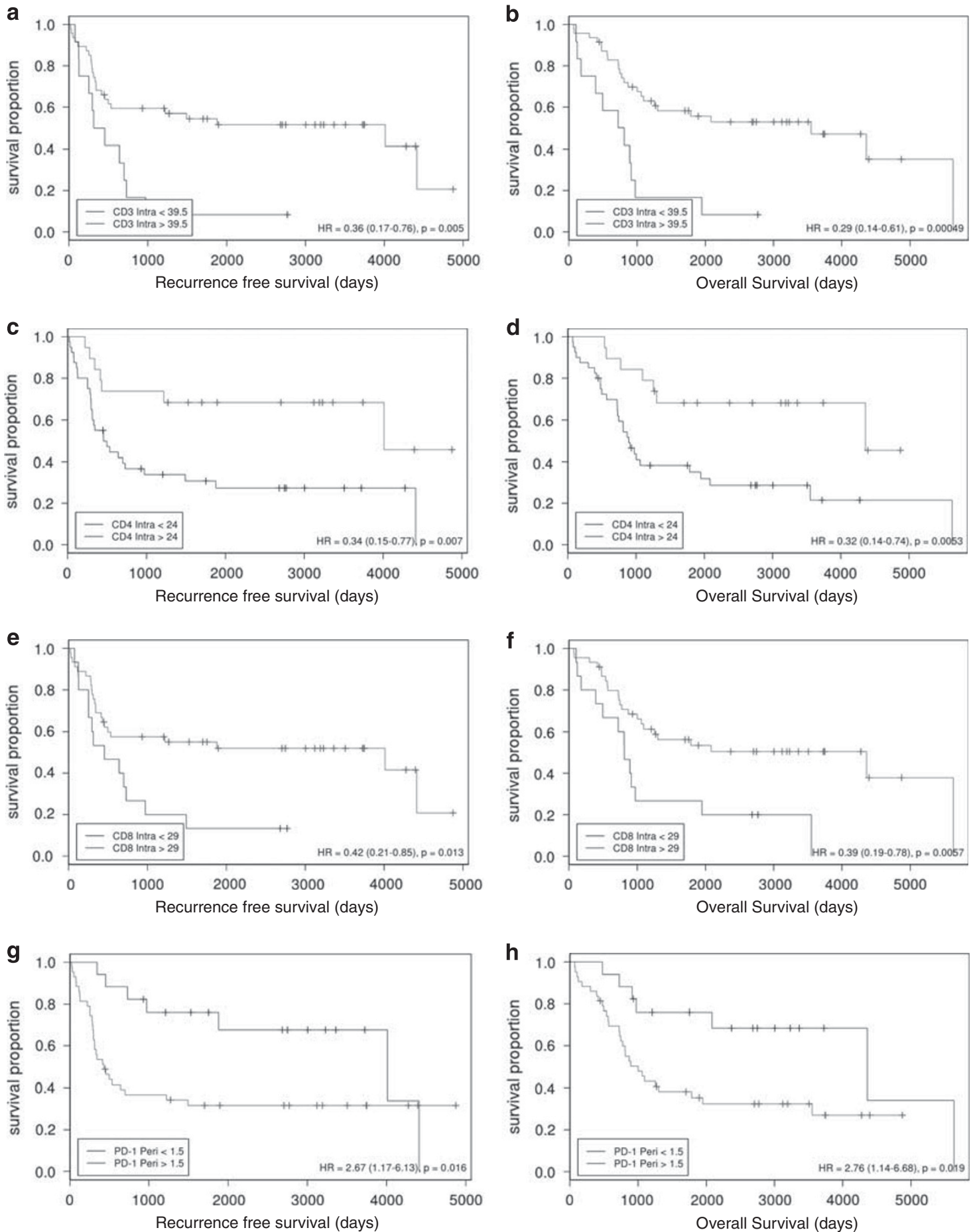

Figure 3 Univariate Cox regression modeling showed that the recurrence-free survival (days) and overall survival (days) of metastatic melanoma-positive sentinel lymph node patients was positively associated with higher $(>39.5,>24,>29$, respectively) intratumoral (intra) CD3+ lymphocyte count (a and b), CD4+ lymphocyte count (c and $\mathbf{d}$ ), and CD8+ lymphocyte count (e and f). There was also a negative association seen in the same patients with higher $(>1.5)$ peritumoral (peri) PD-1+ lymphocyte count (g and h). Inserts demonstrate the optimal cutoff used for each of the markers to separate high and low lymphocyte counts (Significance taken at $P<0.05$ ).

also suggests that the PD-1/PD-L1 axis may be contributing to disease progression in patients with larger sentinel lymph node metastases. Tumor PD-L1 can also be expressed in a constitutive manner, yet this only accounts for less than $5-10 \%$ of tumor PD-L1 positive cases ${ }^{25,29}$ and these patients could potentially gain the greatest benefit from specific PD-L1 inhibitors, which show a significant association between tumor or immune cell PD-L1 expression and response to anti-PD-L1 inhibitors. ${ }^{35}$ 
The prognostic significance of tumor-infiltrating lymphocyte grade in primary cutaneous melanomas $\geq 0.75 \mathrm{~mm}$ thickness has been demonstrated previously and it has been shown that tumor-infiltrating lymphocytes can provide important prognostic information in a multivariate model with the presence of age, ulceration, tumor thickness and mitotic rate. ${ }^{36,37}$ In one study, the presence of tumorinfiltrating lymphocytes in lymph node melanoma metastases predicted recurrence-free survival in multivariate analysis with significantly improved responses seen in patients treated with adjuvant interferon therapy. ${ }^{38}$ In our study we found that the presence of high intratumoral CD4+ helper T cells and CD8+ cytotoxic T cells in sentinel lymph node melanoma metastases predicted for recurrencefree survival and overall survival. To the best of our knowledge, prognostic associations of tumorinfiltrating lymphocytes and tumor-infiltrating lymphocyte subsets in sentinel lymph node metastases from melanoma patients have not previously been reported. Furthermore, the negative association between the presence of peritumoral PD-1+ differentiated lymphocytes and both recurrence-free survival and overall survival has not been previously reported in untreated sentinel lymph node positive melanoma patients and could underpin one of the mechanisms of immune resistance that melanoma cells employ to overcome eradication in the hostile microenvironment of the sentinel lymph node. The overexpression of FOXP3+ regulatory $\mathrm{T}$ cells in lymph nodes containing metastatic melanoma samples has been shown to inhibit the function of infiltrating CD4+ helper and CD8+ cytotoxic T cells through a cell-contact dependent mechanism, ${ }^{39}$ which supports the trend in our study of an increased hazard ratio $>2$ for recurrence-free survival and overall survival in patients with higher peritumoral FOXP3+ lymphocytes. It is possible that this association failed to reach significance due to the relatively low number of uncensored patients in this cohort $(n=36)$, and multivariate analysis including tumor thickness, mitotic rate and ulceration of the primary tumor needs to be undertaken on a larger set of samples.

In conclusion, the frequent presence of tumoral PD-L1 expression in metastatic melanoma deposits in sentinel lymph nodes and the immune microenvironment establishes a scientific rationale for administering adjuvant immune checkpoint inhibitor therapy with either anti-PD1/PD-L1 inhibitor in AJCC stage IIIA sentinel lymph node positive melanoma patients with the potential for improving clinical outcomes. Furthermore, our data also demonstrated the prognostic significance of tumorinfiltrating lymphocytes and lymphocyte subsets in sentinel lymph node positive melanoma patients, which need to be taken into account when design, conduct and analysis of clinical trials assessing the efficacy of immune therapies targeted to tumorinfiltrating lymphocytes. It also has the potential for identifying those patients that will receive the greatest benefit from this treatment regimen.

\section{Acknowledgments}

This work was supported by the National Health and Medical Research Council of Australia (NHMRC), Cancer Institute New South Wales and Melanoma Institute Australia. The authors thank members of the Melanoma Institute Australia including Jonathan Stretch, Robyn Saw, Andrew Spillane, Kerwin Shannon, Valerie Jakrot, Rosa Dobney, Chitra De Silva, Jason Madore, Elizabeth Smith, Ricardo De Paoli, and Elisa James for their support in conducting this project. HK is supported by the Australian Postgraduate Awards (APA) scholarship and a top-up scholarship from the Melanoma Institute Australia distributed through the University of Sydney.

\section{Disclosure/conflict of interest}

GVL is a consultant advisor to Amgen, BMS, GSK, Novartis, Provectus, Roche, and has received Honoraria from BMS, Roche, and GSK. JHY is an employee of Merck. JFT has been an Advisory Board member and received Honoraria from GSK, BMS and Provectus. The remaining authors declare no conflicts of interest.

\section{References}

1 Morton DL, Thompson JF, Cochran AJ et al. Final trial report of sentinel-node biopsy versus nodal observation in melanoma. New Engl J Med 2014;370:599-609.

2 Balch CM, Gershenwald JE, Soong S-j et al. Final version of 2009 AJCC melanoma staging and classification. J Clin Oncol 2009 27:6199-6206.

3 Eggermont AM, Suciu S, Santinami M et al. Adjuvant therapy with pegylated interferon alfa-2b versus observation alone in resected stage III melanoma: final results of EORTC 18991, a randomised phase III trial. Lancet 2008;372:117-126.

4 Mocellin S, Pasquali S, Rossi CR et al. Interferon alpha adjuvant therapy in patients with high-risk melanoma: a systematic review and meta-analysis. J Nat Cancer I 2010;102:493-501.

5 Kirkwood JM, Strawderman MH, Ernstoff MS et al. Interferon alfa-2b adjuvant therapy of high-risk resected cutaneous melanoma: the Eastern Cooperative Oncology Group Trial EST 1684. J Clin Oncol 1996;14:7-17.

6 Kirkwood JM, Manola J, Ibrahim J et al. A pooled analysis of eastern cooperative oncology group and intergroup trials of adjuvant high-dose interferon for melanoma. Clin Cancer Res 2004;10:1670-1677.

7 Hodi FS, O’Day SJ, McDermott DF et al. Improved survival with ipilimumab in patients with metastatic melanoma. New Engl J Med 2010;363:711-723.

8 Chapman PB, Hauschild A, Robert C et al. Improved survival with vemurafenib in melanoma with BRAF V600E mutation. New Engl J Med 2011;364:2507-2516. 
9 Hauschild A, Grob J-J, Demidov LV et al. Dabrafenib in BRAF-mutated metastatic melanoma: a multicentre, open-label, phase 3 randomised controlled trial. Lancet 2012;380:358-365.

10 Long GV, Stroyakovskiy D, Gogas H et al. Combined BRAF and MEK inhibition versus BRAF inhibition alone in melanoma. New Engl J Med 2014;371: 1877-1888.

11 Robert C, Karaszewska B, Schachter J et al. Improved overall survival in melanoma with combined dabrafenib and trametinib. New Engl J Med 2015;372:30-39.

12 Larkin J, Ascierto PA, Dréno B et al. Combined vemurafenib and cobimetinib in BRAF-mutated melanoma. New Engl J Med 2014;371:1867-1876.

13 Topalian SL, Hodi FS, Brahmer JR et al. Safety, activity, and immune correlates of anti-PD-1 antibody in cancer. New Engl J Med 2012;366:2443-2454.

14 Hamid O, Robert C, Daud A et al. Safety and Tumor Responses with Lambrolizumab (Anti-PD-1) in Melanoma. New Engl J Med 2013;369:134-144.

15 Ishida Y, Agata Y, Shibahara K et al. Induced expression of PD-1, a novel member of the immunoglobulin gene superfamily, upon programmed cell death. EMBO J 1992;11:3887.

16 Keir ME, Butte MJ, Freeman GJ et al. PD-1 and its ligands in tolerance and immunity. Annu Rev Immunol 2008;26:677-704.

17 Freeman GJ, Long AJ, Iwai Y et al. Engagement of the PD-1 immunoinhibitory receptor by a novel B7 family member leads to negative regulation of lymphocyte activation. J Exp Med 2000;192:1027-1034.

18 Dong H, Zhu G, Tamada K et al. B7-H1, a third member of the B7 family, co-stimulates T-cell proliferation and interleukin-10 secretion. Nat Med 1999;5:1365-1369.

19 Latchman Y, Wood CR, Chernova T et al. PD-L2 is a second ligand for PD-1 and inhibits $\mathrm{T}$ cell activation. Nat Immunol 2001;2:261-268.

20 Tseng S-Y, Otsuji M, Gorski $\mathrm{K}$ et al. B7-DC, a new dendritic cell molecule with potent costimulatory properties for T cells. J Exp Med 2001;193: 839-846.

21 Yamazaki $\mathrm{T}$, Akiba $\mathrm{H}$, Iwai $\mathrm{H}$ et al. Expression of programmed death 1 ligands by murine $\mathrm{T}$ cells and APC. J Immunol 2002;169:5538-5545.

22 Iwai Y, Ishida M, Tanaka Y et al. Involvement of PD-L1 on tumor cells in the escape from host immune system and tumor immunotherapy by PD-L1 blockade. Proc Natl Acad Sci USA 2002;99:12293-12297.

23 Dong H, Strome SE, Salomao DR et al. Tumor-associated B7-H1 promotes T-cell apoptosis: a potential mechanism of immune evasion. Nat Med 2002;8:793-800.

24 Wilmott JS, Long GV, Howle JR et al. Selective BRAF inhibitors induce marked T-cell infiltration into human metastatic melanoma. Clin Cancer Res 2012;18: 1386-1394.

25 Kakavand H, Wilmott JS, Menzies AM et al. PD-L1 Expression and Tumor-Infiltrating Lymphocytes Define Different Subsets of MAPK Inhibitor-Treated Melanoma Patients. Clin Cancer Res 2015;21:3140-3148.

26 Tumeh PC, Harview CL, Yearley JH et al. PD-1 blockade induces responses by inhibiting adaptive immune resistance. Nature 2014;515:568-571.
27 Morton DL, Thompson JF, Cochran AJ et al. Sentinelnode biopsy or nodal observation in melanoma. New Engl J Med 2006;355:1307-1317.

28 Eggermont AM (ed). Ipilimumab versus placebo after complete resection of stage III melanoma: Initial efficacy and safety results from the EORTC 18071 phase III trial. Proceedings of the ASCO Annual Meeting; 30 May-03 June 2014; Chicago, IL, USA. American Society of Clinical Oncology: Chicago, IL, USA, 2014.

29 Madore J, Vilain RE, Menzies AM et al. PD-L1 expression in melanoma shows marked heterogeneity within and between patients: implications for antiPD-1/PD-L1 clinical trials. Pigm Cell Mel Res 2014;28: 245-253.

30 Taube JM, Anders RA, Young GD et al. Colocalization of inflammatory response with B7-h1 expression in human melanocytic lesions supports an adaptive resistance mechanism of immune escape. Sci Transl Med 2012;4:127ra37-1ra37r.

31 Gadiot J, Hooijkaas AI, Kaiser ADM et al. Overall survival and PD-L1 expression in metastasized malignant melanoma. Cancer 2011;117:2192-2201.

32 Robert C, Long GV, Brady B et al. Nivolumab in previously untreated melanoma without BRAF mutation. New Engl J Med 2015;372:320-330.

33 Kefford R (ed). Clinical efficacy and correlation with tumor PD-L1 expression in patients (pts) with melanoma (MEL) treated with the anti-PD-1 monoclonal antibody MK-3475. Proceedings of the ASCO Annual Meeting; 30 May-03 June 2014; Chicago, IL, USA. American Society of Clinical Oncology: Chicago, IL, USA, 2014.

34 Herbst RS (ed). A study of MPDL3280A, an engineered PD-L1 antibody in patients with locally advanced or metastatic tumors. Proceedings of the ASCO Annual Meeting; 31 May-04 June 2013; Chicago, IL, USA. American Society of Clinical Oncology: Chicago, IL, USA, 2013.

35 Herbst RS, Soria J-C, Kowanetz M et al. Predictive correlates of response to the anti-PD-L1 antibody MPDL3280A in cancer patients. Nature 2014;515: 563-567.

36 Azimi F, Scolyer RA, Rumcheva $\mathrm{P}$ et al. TumorInfiltrating Lymphocyte Grade Is an Independent Predictor of Sentinel Lymph Node Status and Survival in Patients With Cutaneous Melanoma. J Clin Oncol 2012;30:2678-2683.

37 Clemente CG, Mihm MC, Bufalino R et al. Prognostic value of tumor infiltrating lymphocytes in the vertical growth phase of primary cutaneous melanoma. Cancer 1996;77:1303-1310.

38 Mihm MC Jr, Clemente CG, Cascinelli N. Tumor infiltrating lymphocytes in lymph node melanoma metastases: a histopathologic prognostic indicator and an expression of local immune response. Lab Invest 1996;74:43-47.

39 Viguier M, Lemaître F, Verola O et al. Foxp3 expressing CD4+ CD25high regulatory $\mathrm{T}$ cells are overrepresented in human metastatic melanoma lymph nodes and inhibit the function of infiltrating $\mathrm{T}$ cells. J Immunol 2004;173:1444-1453. 